\title{
Query Answer Reformulation over Knowledge Bases
}

\author{
João Pedro V. Pinheiro' ${ }^{1}$, Marco A. Casanova ${ }^{1}$, Elisa S. Menendez ${ }^{2}$ \\ 1 Department of Informatics, PUC-Rio \\ 2 Federal Institute of Education, Science and Technology Baiano \\ \{jpinheiro, casanova\}@inf.puc-rio.br, elisa.menendez@ifbaiano.edu.br
}

\begin{abstract}
The answer of a query, submitted to a database or a knowledge base, is often long and may contain redundant data. The user is frequently forced to browse through a long answer or refine and repeat the query until the answer reaches a manageable size. Without proper treatment, consuming the answer may indeed become a tedious task. This article then proposes a process that modifies the presentation of a query answer to improve the quality of the user's experience in the context of an RDF knowledge base. The process reorganizes the original query answer by applying heuristics to summarize the results and to select template questions that create a user dialog that guides the presentation of the results. The article also includes experiments based on RDF versions of MusicBrainz, enriched with DBpedia data, and IMDb, each with over 200 million RDF triples. The experiments use sample queries from well-known benchmarks.
\end{abstract}

Categories and Subject Descriptors: H.2 [Database Management]: Heterogeneous Databases; H.3 [Information Storage and Retrieval]: Information Search and Retrieval

Keywords: Aggregation, Summarization, Natural Language Query (NLQ), Question Answering (QA), RDF, Semantic Web

\section{INTRODUCTION}

Question Answering (QA) systems combine techniques from multiple fields of computer science, including Natural Language Processing (NLP), Information Retrieval, Machine Learning (ML), and Semantic Web. Assuming that the user is interested in querying a database or a knowledge base, a QA system may be split into two parts: question, which receives a user's input in natural language, transforms it into a structured query and searches the data; and answer, which displays consistent results in a human-readable format to the user. The answer to a query is often long and may contain redundant data. The user is frequently forced to browse through a long answer or refine and repeat the query until the answer reaches a manageable size. Without proper treatment, consuming the answer may indeed become a tedious task.

This article addresses the problem of query answer modification to improve the quality of the user's experience, in the context of an RDF knowledge base. For example, imagine yourself as a user interacting with a virtual voice assistant, and you ask an open-ended question about a specific subject, e.g., "Which artists were born on May $30^{\text {th }}$ ?". The query answer may have a long list of artists, partly shown in Table I. Instead of listing the results, the virtual assistant may formulate questions to the user based on the prior result set, such as: "Do you want to list American or European artists?"; "Do you prefer Jazz, Pop, or Classical music?"; and "Do you want to filter by active artists?".

The article proposes a process that reorganizes the original query answer by applying heuristics to summarize the results and to select template questions that create a user dialog that guides the presentation of the results. The heuristics allow deciding which properties returned in the query

Copyright(C)2021 Permission to copy without fee all or part of the material printed in JIDM is granted provided that the copies are not made or distributed for commercial advantage, and that notice is given that copying is by permission of the Sociedade Brasileira de Computação. 
Table I. Example of question-answer for an open-ended question.

\begin{tabular}{|l|l|l|l|l|l|}
\hline \multicolumn{1}{|c|}{ Artist } & Genre & Birth Date & Death Date & Gender & Nationality \\
\hline Goodman, Benny & Jazz & $1909-05-30$ & $1986-06-13$ & Male & American \\
\hline Leonhardt, Gustav & Classical & $1928-05-30$ & $2012-01-16$ & Male & Dutch \\
\hline Green, CeeLo & Pop & $1974-05-30$ & & Male & American \\
\hline Biosphere & Eletronic & $1962-05-30$ & & Male & Norwegian \\
\hline Fredriksson, Marie & Pop & $1958-05-30$ & $2019-12-09$ & Female & Swedish \\
\hline Banhart, Devendra & Folk & $1981-05-30$ & & Male & American \\
\hline
\end{tabular}

answer are interesting to apply aggregations (group by operations) and which template questions best fit each case. The heuristics also help decide if the answer is ready to be displayed to the user, or if the answer must be improved. The decisions use global statistics about the RDF dataset, obtained a priori, and local statistics about the query answer, obtained dynamically. The statistics are related to the frequency of the class instances and the frequency of the predicates.

The article also includes experiments based on the RDF versions of MusicBrainz and IMDb described in [Menendez et al. 2019], and obtained by enriching a MusicBrainz dump with DBpedia data and transforming an IMDb relational database to RDF via R2RML, respectively. Each RDF dataset has over 200 million triples. Our experiments use sample queries from the QALD - Question Answering over Linked Data ${ }^{1}$ challenge and from Coffman's benchmark [Coffman and Weaver 2010].

The rest of the paper is organized as follows. Section 2 summarizes related work. Section 3 discusses the query answer modification process. Section 4 describes the experiments. Finally, Section 5 presents the conclusions and directions for future research.

\section{RELATED WORK}

In a seminal paper, Webber proposed a theoretical framework that divided the communication between humans and machines into three parts [Webber 1986]. Instead of only considering questions and answers, Webber proposed a clear distinction between a question, an answer, and a response. A question is a request by a user that demands information or asks to perform an action. An answer is the information or performance directly requested. A response embraces multiple elements, such as a direct answer, information or actions related to the original request instead of an answer, and additional information or actions when no proper answer can be found (also called "did you mean?"). Comparing the proposed framework with our work, we consider only open-ended questions. Thus, we can assume that a question is strictly demanding information. From the point of view of the answer (called "response" in the framework), our process suggests information related to the original request instead of a single answer. This information is presented to the user as facets, which allows constructing a dialog and providing better guidance to the desired answer.

Knowledge Base systems are usually constructed from multiple sources, leading to the generation of duplicated data. By contrast, humans avoid redundancy in the act of writing or speaking. Indeed, reducing duplicated data in the communication between humans and machines is a challenging task. Aggregation and summarization are important techniques that help to solve this issue.

The problem of redundancy is addressed, for example, in [Dalianis and Hovy 1996]. The authors suggested aggregation strategies to remove redundancy from the text - usually retrieved answers from databases. An interesting example, used in the paper, to illustrate the problem goes as follows. Consider the question:

\footnotetext{
"Who is currently at ISI?",
}

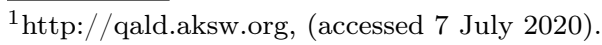


Suppose that the answer to this question is:

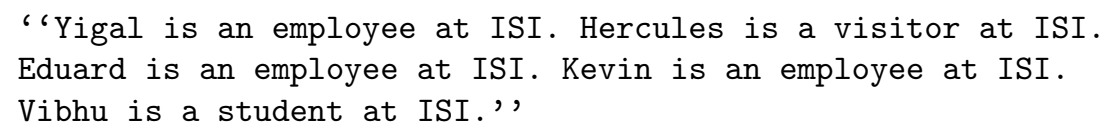

Note that the answer is too long and repetitive. After applying the aggregation rules suggested in the paper, the modified answer is shortened and easier to understand:

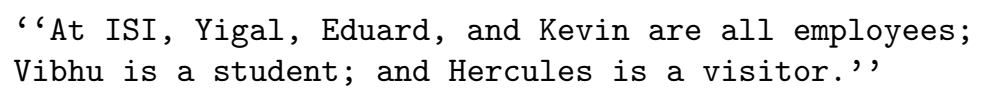

The authors developed a questionnaire and applied it to computer scientists. The questionnaire was composed of five example sets of input data, and each example contained between 11 and 18 propositions. After analyzing the answers, four classes or types of aggregation rules became obvious: grouping and collapsing rules, ordering rules, casting rules, and parsimony rules. Furthermore, the paper listed eight aggregation strategies, each related to one aggregation class. As future work, the authors suggested a follow-up study involving a larger group of people, not all of whom being computer scientists, for more general results. Also, in the last section of the paper, the authors presented some scenarios that lead to unsatisfactory results and listed future improvements.

Deutch et al. (2017) described an approach to present query results as sentences in Natural Language (NL) with provenance information. The authors argued that the answers in the query result lack justification and suggested the notion of provenance, which corresponds to including additional information to the query results. Also, provenance information helps validate answers. The paper used the MAS (Microsoft Academic Research) publication database to validate the results. The proposed solution had the following key contributions: provenance tracking based on the NL query structure, factorization, summarization, and implementation and experiments.

An important step was provenance tracking based on the query structure. Deutch et al. (2017) used two external tools in this process. The modified NaLIR (Natural Language Interface for Relational databases) tool was used to store exactly which parts of an NL query translate to which parts of the formal query. The evaluation of the formal query used the provenance-aware engine SelP - Selective tracking and presentation of data provenance. It stored which parts of the query "contributed" to which parts of the provenance. Thus, two mappings were available for the next steps: text-to-query-parts and query-parts-to-provenance.

This study was the first one to address provenance for the NL queries problem. After implementation and experiments, the authors listed two main limitations of their work. The sentence generation module was specifically designed for NaLIR and will need to be replaced if a different NL query engine is used. Second, the solution is limited to conjunctive queries, not supporting unions and aggregations.

In a similar direction, faceted browsing [Petzka et al. 2017] (also called "faceted search" [Wei et al. 2013]) is a complement to keyword search, which provides an iterative way to refine search results. Facets are usually displayed to the user as rectangles right next to the main list of results provided by keyword search. These facets contain relevant grouped information which guides users to the desired answer.

In [Moreno-Vega and Hogan 2018] and [Franz et al. 2009], faceted browsing was used to simplify the user's interaction with data. Moreno-Vega and Hogan (2018) allowed search by keyword or type. A type would be an IRI from the RDF graph. While there are facets available, the user can navigate interacting only with them. Facets with zero results are never offered. Franz et. al. (2009) used faceted browsing for user evaluation purposes. With fixed subject and predicate, the user receives a 
list of objects sorted by the so-called TripleRank and decides if each of the objects "is related", "does not know", or "is not related".

Finally, several studies addressed the problem of creating a Natural Language interface to databases, such as [Novello and Casanova 2020]. Usually, the proposed process has four steps: Question Analysis, Phrase Mapping, Disambiguation, and Query Construction - not necessarily in this order [Diefenbach et al. 2018]. In this article, we assume that the Natural Language interface is constructed over an RDF knowledge base and accessed through a SPARQL endpoint.

\section{THE QUERY ANSWER MODIFICATION PROCESS}

This section is organized as follows. Section 3.1 provides some basic definitions required for our discussion. Section 3.2 describes the process of transforming a single-column into a three-column result set. Section 3.3 addresses the use of frequency analysis based on RDF metadata. Section 3.4 briefly discusses how to construct the user dialog.

\subsection{Basic Definitions}

The Resource Description Framework - RDF [Cyganiak et al. 2014] is a standard model for data interchange on the Web. A key characteristic of RDF is the ability to model data and metadata without distinction, which facilitates the evolution of schemes over time without impairing the way data are consumed. An RDF triple is of the form $(s, p, o)$, where $s$ is the subject, $p$ is the predicate, and $o$ is the object of the triple. An RDF triple set (also called an RDF dataset) is a set of RDF triples, which induces a directed, labeled graph, whose nodes are the subjects and objects of the triples and there is an edge labeled with $p$ iff there is a triple $(s, p, o)$ in the triple set.

SPARQL is the standard query language for RDF [Prud'hommeaux and Seaborne 2008]. A simple example of a SPARQL query, which retrieves the URIs of all movies performed by "Denzel Washington", is:

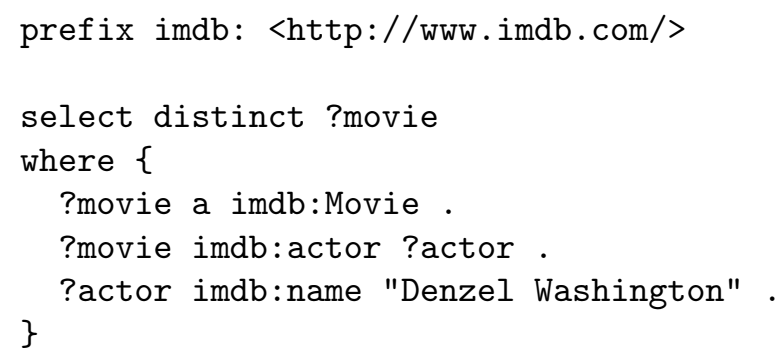

The query form select followed by the solution modifier distinct guarantees that only unique URIs will be presented in the result set. The where clause restricts the result by applying a graph pattern matching over the RDF graph. Also the term ' $a$ ' is the syntactical sugar form of the predicate rdf : type.

SPARQL also supports aggregation and subqueries, which are the main topics of this article, addressed in Section 3.2.

\subsection{Transforming single-column into three-column result sets}

The query answer modification process we propose starts after the query is executed. The expected inputs are the SPARQL query and the result set, as illustrated in Figure 1. There are two possible scenarios: the result set has a single column, or the result set has multiple columns. Our study focuses on the first case. 


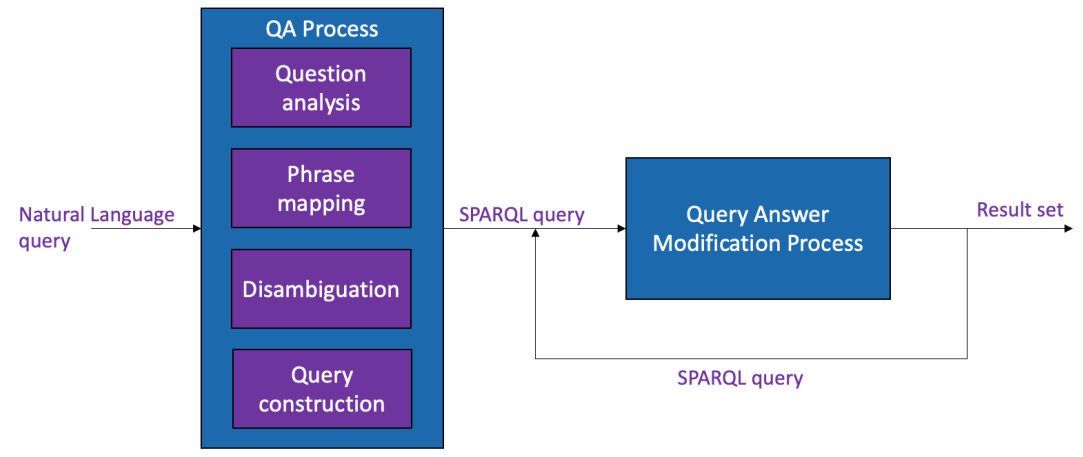

Fig. 1. Query answer modification process.

We base our discussion on a series of question-answering challenges over Linked Data, referred to as QALD - Question Answering over Linked Data ${ }^{2}$. Several papers use QALD to measure quality metrics of the system's answers [Diefenbach et al. 2017]. We noticed that most queries listed in the QALD challenges had single-column answers, which calls for enriching the answers for the purposes of this paper. A simple approach is to add to the instances returned their property values. Indeed, frequently, the answers represent sets of instances of the same rdf:type. So, it is straightforward to modify the original SPARQL query to also retrieve the desired property values.

As an example, consider again the question "Which artists were born on May $30^{\text {th }}$ ?". The result set of the corresponding SPARQL query has instances of type mo:MusicArtist, as in Figure 2(c). Then, modifying the original SPARQL query also enables to retrieve property values, as shown in Figure 2(d). Note that, in Figure 2(d), the column artist has repeated values. However, instead of normalizing the returned table, we decided to keep this three-column format to simplify data manipulation.

\subsection{Frequency analysis based on computed metadata}

In the process we propose, a set of SPARQL queries is used to generate graph statistics, which help decide what to do next. These statistics are related to the frequency of the instances by class and the frequency of the predicates.

There are two types of frequencies used. A global frequency is defined over the full graph and is computed only once before any query is executed. On the other hand, a local frequency is defined over the sub-graph generated as in Section 3.2 and is computed at run time. Both global and local frequencies are computed over predicates pointing to literals only.

Entity ranking is based on InfoRank, a family of importance measures proposed in [Menendez et al. 2019]. The proposed importance measures are combinations of three intuitions: (I) "important things have lots of information about them"; (II) "important things are surrounded by other important things"; (III) "few important relations (e.g., friends) are better than many unimportant relations (e.g. acquaintances)". Hence, the strategy is based on the level of informativeness of an entity, represented as literals in RDF graphs. They use a PageRank-inspired approach to propagate the importance scores from entity to entity. The InfoRank metric helps our process prioritize the most relevant triples of the result set.

As an example, Figure 3 shows an instance A1. The initial state (Figure 3(a)) has predicates pointing to literals and other instances. Notice that the final state (Figure 3(b)) only has predicates pointing to literals and an extra predicate called InfoRank.

\footnotetext{
${ }^{2}$ http://qald.aksw.org, (accessed 7 July 2020). 


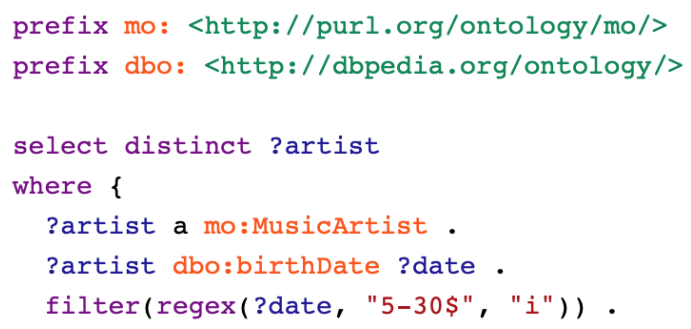

(a) Single-column query

\begin{tabular}{|l|}
\hline \multicolumn{1}{c|}{ artist } \\
\hline mo:MusicArtist/1 \\
\hline mo:MusicArtist/2 \\
\hline mo:MusicArtist/3 \\
\hline mo:MusicArtist/4 \\
\hline mo:MusicArtist/5 \\
\hline mo:MusicArtist/6 \\
\hline mo:MusicArtist/7 \\
\hline mo:MusicArtist/8 \\
\hline mo:MusicArtist/9 \\
\hline mo:MusicArtist/10 \\
\hline
\end{tabular}

(c) Single-column result set

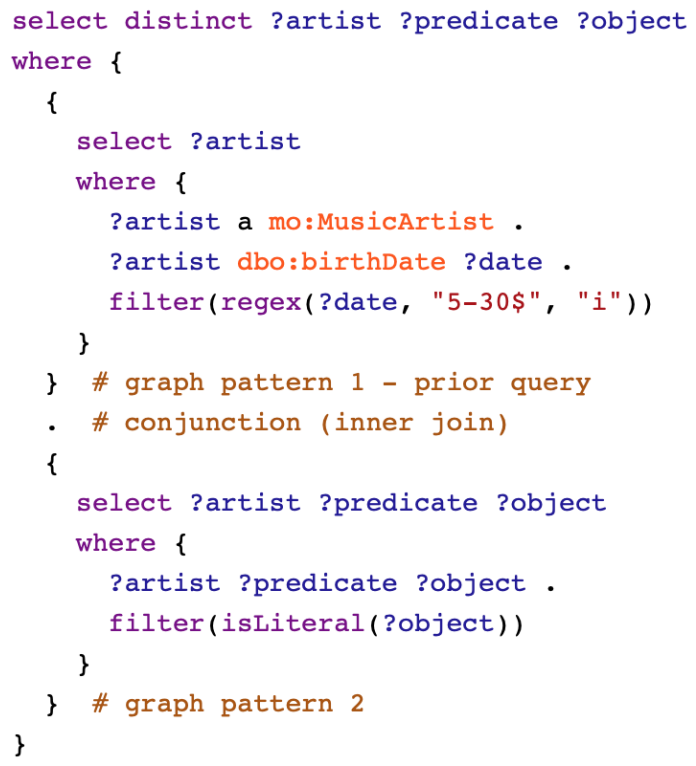

(b) Three-column query

\begin{tabular}{|l|l|l|}
\hline \multicolumn{1}{|c|}{ artist } & \multicolumn{1}{|c|}{ predicate } & \multicolumn{1}{c|}{ object } \\
\hline mo:MusicArtist/1 & foaf : name & "Green, CeeLo" \\
\hline mo:MusicArtist/1 & mo: genre & "pop" \\
\hline mo:MusicArtist/1 & dbo: BirthDate & "1974-05-30" \\
\hline mo:MusicArtist/1 & dbo: DeathDate & " " \\
\hline mo:MusicArtist/1 & foaf : gender & "Male" \\
\hline mo:MusicArtist/1 & dbp: nationality & "American" \\
\hline mo:MusicArtist/2 & foaf: name & "Leonhardt, Gustav" \\
\hline mo:MusicArtist/2 & mo: genre & "Classical" \\
\hline mo:MusicArtist/2 & dbo: BirthDate & "1928-05-30" \\
\hline mo:MusicArtist/2 & dbo: DeathDate & "2012-01-16" \\
\hline mo:MusicArtist/2 & foaf: gender & "Male" \\
\hline mo:MusicArtist/2 & dbp: nationality & "Dutch" \\
\hline mo:MusicArtist/3 & foaf: name & "Goodman, Benny" \\
\hline mo:MusicArtist/3 & mo: genre & "Jazz" \\
\hline mo:MusicArtist/3 & dbo: BirthDate & "1909-05-30" \\
\hline mo:MusicArtist/3 & dbo: DeathDate & "1986-06-13" \\
\hline mo:MusicArtist/3 & foaf: gender & "Male" \\
\hline mo:MusicArtist/3 & dbp: nationality & "American" \\
\hline
\end{tabular}

(d) Three-column result set

Fig. 2. Transformation with SPARQL queries.

Parameterized thresholds are used to filter predicates that are candidates to be used in a group_by operation. By default, these threshold values $(\delta)$ are set between 0.4 and 2.0, which means the predicate must appear in at least $40 \%$ and not more than $200 \%$ of the unique subjects. For clarity, consider again the question "Which artists were born on May $30^{\text {th }}$ ?". Analyzing the three-column result set, the predicate rdfs:comment appears 497 times and there are 123 unique artists. This means the predicate appears 4.04/artist on average. Thus, the predicate rdfs:comment is removed by the process because it exceeds the maximum threshold. 


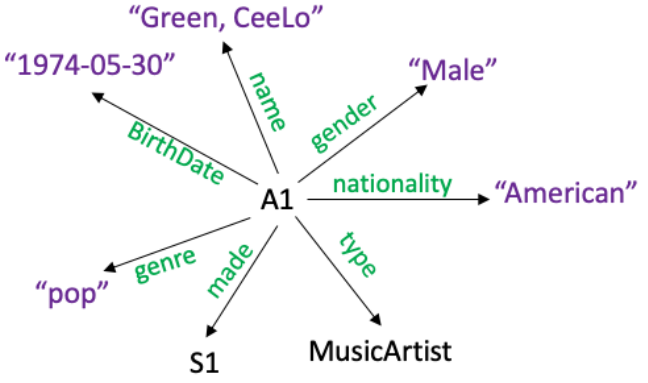

(a) All predicates

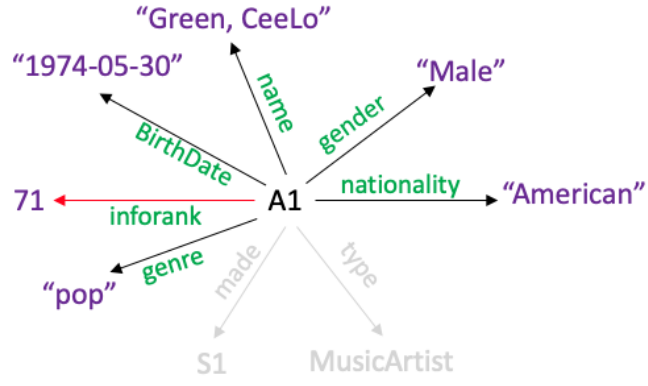

(b) Filtered predicates

Fig. 3. Filtered predicates by literal and highlighted InfoRank.

\subsection{Computing the user dialog}

As mentioned in the previous section, an aggregation process is applied over the filtered predicates. These predicates are evaluated and sorted by their local frequency. Another threshold is related to the number of aggregated values $(\alpha)$, which has 10 as the default value. After this filter, the process chooses which template question must be used and returns a single new question to the user.

For example, consider once again the question "Which artists were born on May 30 ${ }^{\text {th }}$ ?". Analyzing the previous filtered candidates, the predicate dbo:activeYearsStartYear has 35 unique values. Although it might be a promising candidate, the predicate dbo:activeYearsStartYear is removed by the process because it exceeds the $\alpha$ threshold.

Template questions are choice questions, formulated in natural language, that offer aggregated predicates as alternatives to the user. Using the same example, the filtered predicates are: foaf : gender, with two aggregated values - female and male; and dbo:background, with three aggregated values - non_performing_personnel, non_vocal_instrumentalist, and solo_singer. Thus, the process may generate the following questions:

(1) Between \{non_performing_personnel\}, \{non_vocal_instrumentalist $\}$, and $\{$ solo_singer $\}$, which artists do you prefer?

(2) Do you prefer $\{$ female $\}$, or $\{$ male $\}$ artists?

Since dbo:backgroud precedes foaf:gender in the computed frequencies, only question (1) is returned to the user. If the user keeps interacting with the system, the whole process restarts. Also, there is a final threshold $(\beta)$ responsible for limiting the number of elements returned to the user, which has 15 as the default value.

\section{EXPERIMENTS}

\subsection{Setup}

We performed initial experiments using the RDF versions of MusicBrainz and $\mathrm{IMDb}^{3}$ described in [Menendez et al. 2019], obtained by enriching a MusicBrainz dump with DBpedia data and transforming an IMDb relational database to RDF via R2RML, respectively. These datasets also contain the InfoRank scores of instances, properties and classes. Figure 4 shows the resulting MusicBrainz and IMDb schemas, respectively. Each RDF dataset has over 200 million triples. We used sample queries from the QALD challenge and Coffman's benchmark [Coffman and Weaver 2010].

\footnotetext{
${ }^{3}$ https://sites.google.com/view/quira/, (accessed 7 July 2020). 

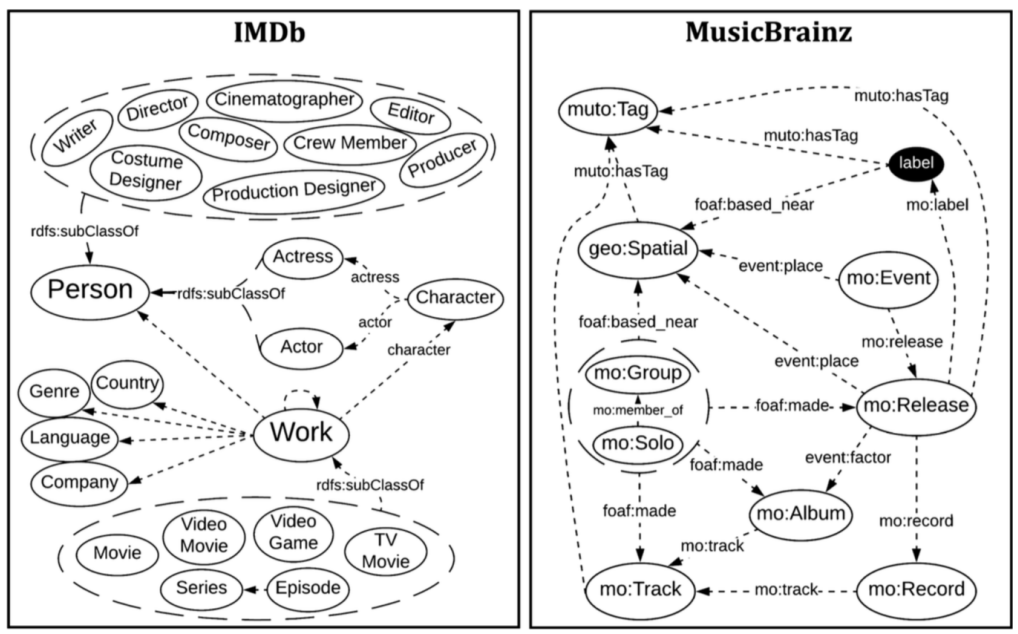

Fig. 4. IMDb and MusicBrainz schemas.

To store and manage the RDF datasets, we used the component TDB2 of Apache Jena for RDF ${ }^{4}$. Apache Jena Fuseki (a SPARQL server) ran on a server machine with OS GNU/Linux Ubuntu 16.04.6 LTS, a quad-core processor Intel(R) Core(TM) i7-5820K CPU @ 3.30GHz, 64 GB of RAM and SSD $1 \mathrm{~TB}$.

In the following sections, we discuss the effect of the proposed query reformulation process - referred to as the process, for brevity - over both datasets on the query result. The thresholds used for these experiments were: $\alpha=10, \beta=15$ and $\delta=\left(\delta_{\min }, \delta_{\max }\right)=(0.4,2.0)$.

\subsection{MusicBrainz Results}

In this section, we detail the experiments with the QALD query for MusicBrainz presented earlier: "Which artists were born on May $30^{\text {th }}$ ?". The initial process generated the following SPARQL query, with results ranked by the InfoRank score.

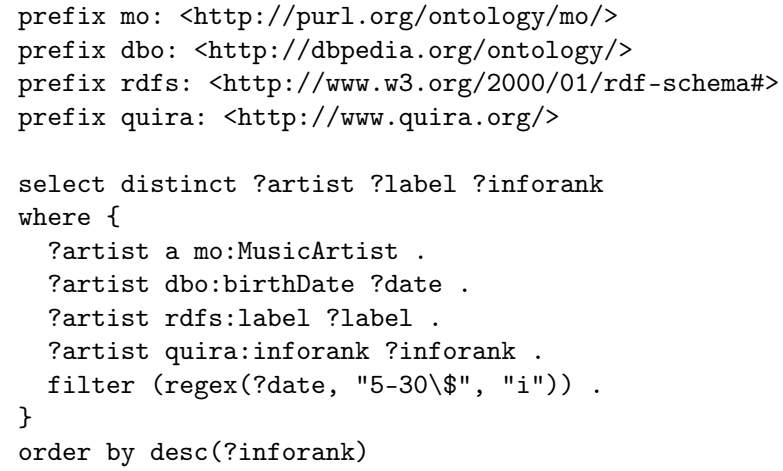

Table II shows a preview of the original result. Note that the SPARQL query returned 122 artists that were born on May $30^{t h}$, which the user might consider to be a long list to interact with. We defined a threshold to indicate when the (reformulation) process should be applied. For these experiments, we chose a maximum of $\beta=15$ lines, that is, a list with only $\beta$ artists in this example. Hence, the

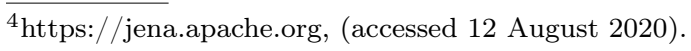


process reformulated the SPARQL query to capture the predicates that are candidates to be used for aggregation.

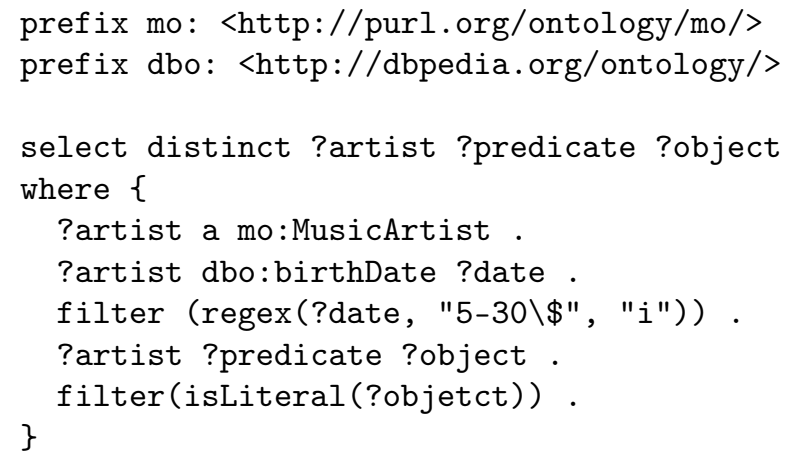

The result was stored in memory to facilitate manipulation and to avoid further access to the database. Then, the process grouped the results by predicate and counted the distinct object values. Table III presents these results.

We defined a threshold of $\alpha$ as the maximum number of distinct values for the predicates, so the questions formulated to the user are not too long. A heuristic is to choose the predicate with the highest number of distinct values from the set of predicates with less distinct values than the maximum. Following this heuristic, the process chose the predicate dbo: background, which refers to the type of music artists. Hence, the process formulated the query "Which of the above artist background do you prefer?" and presented a few options for the user to choose from, as shown in Table IV.

Note that Table IV provides five options to the user: the first three options correspond to the three distinct values of the predicate dbo:background; the fourth option to artists that have some background defined (not all artists have the background defined in the database); and the last option to all artists (artists, without any filter). Suppose the user chose option "1. Solo singer", which has one of the highest artist counts. The final result decreased to 27 artists, as shown in Table V.

Since this result was still higher than our threshold of $\beta=15$ lines, the process was reapplied. Again, the process grouped the results by predicate and counted the distinct object values. Table VI presents this result.

Continuing with the proposed heuristic, the process chose the predicate dbp:occupation and formulated a new question to the user "Which of the above artist occupation do you prefer?" and presented a few options for the user to choose from, as shown in Table VII.

Suppose the user chose option "1. Singer-songwriter, musician" as the artist occupation. The process finally stopped since it achieved our threshold of $\beta$ lines. Hence, the final result was presented to the user in a decreased order of InfoRank score, as shown in Table VIII.

Table II. Preview of the original SPARQL result.

\begin{tabular}{|c|l|l|}
\hline$\#$ & Artist partial URI & Artist name \\
\hline 1 & /artist/b09ae88f-4156-4caa-b129-1cacb5e1632e & Benny Goodman \\
\hline 2 & $/$ artist/27b0750a-7318-4075-9470-43b82d454ea0 & Gustav Leonhardt \\
\hline 3 & /artist/2c69465c-0f76-45ce-90a2-1ed0fdacc997 & CeeLo Green \\
\hline$\ldots$ & $\ldots$ & $\ldots$ \\
\hline 120 & /artist/e00871b0-f6b5-41cf-b758-f2f1ea467818 & Frank St. Leger \\
\hline 121 & /artist/09ffe9f4-d54e-4943-8297-4456963f0def & Josephine Preston Peabody \\
\hline 122 & /artist/22b95e86-0749-4df1-ae29-cd5acfe5a285 & Jim Murray \\
\hline
\end{tabular}


Table III. Available predicates in the first reformulation process.

\begin{tabular}{|c|l|c|}
\hline$\#$ & Predicate & Distinct Values \\
\hline 1 & http://xmlns.com/foaf/0.1/name & 144 \\
\hline 2 & http://dbpedia.org/property/birthDate & 131 \\
\hline 3 & http://xmlns.com/foaf/0.1/givenName & 129 \\
\hline 4 & http://www.w3.org/2000/01/rdf-schema\#label & 123 \\
\hline 5 & http://dbpedia.org/ontology/wikiPageID & 120 \\
\hline 6 & http://dbpedia.org/ontology/wikiPageRevisionID & 120 \\
\hline 7 & http://purl.org/dc/terms/description & 91 \\
\hline 8 & http://xmlns.com/foaf/0.1/surname & 87 \\
\hline 9 & http://dbpedia.org/ontology/deathDate & 53 \\
\hline 10 & http://dbpedia.org/ontology/activeYearsStartYear & 35 \\
\hline 11 & http://dbpedia.org/ontology/background & 3 \\
\hline 12 & http://xmlns.com/foaf/0.1/gender & 2 \\
\hline
\end{tabular}

Table IV. Options for "Which of the above artist background do you prefer?".

\begin{tabular}{|l|l|c|}
\hline$\#$ & Options & Counts \\
\hline 1 & Solo singer & 27 \\
\hline 2 & Non vocal instrumentalist & 25 \\
\hline 3 & Non-performing personnel & 5 \\
\hline 4 & Show me all artists with some background & 57 \\
\hline 5 & Show me all artists & 123 \\
\hline
\end{tabular}

Table V. Preview of the SPARQL result filtered by "Solo singer" background.

\begin{tabular}{|c|l|l|}
\hline$\#$ & Artist partial URI & Artist name \\
\hline 1 & /artist/2c69465c-0f76-45ce-90a2-1ed0fdacc997 & CeeLo Green \\
\hline 2 & /artist/0110e63e-0a9b-4818-af8e-41e180c20b9a & Devendra Banhart \\
\hline 3 & /artist/3a0373c0-f9c1-4eb3-9c10-53cc18193b07 & Marie Fredriksson \\
\hline$\ldots$ & $\ldots$ & $\ldots$ \\
\hline 25 & /artist/0de740a2-a651-4d76-9cd5-54912a64070f & Gladys Horton \\
\hline 26 & /artist/be0c5489-92e2-4149-b094-48293606f34b & Brian Fair \\
\hline 27 & /artist/ae148627-23cc-48d3-a1a7-804f2af6b7dc & Rick DePiro (Ricky Dee) \\
\hline
\end{tabular}

\subsection{IMDb Results}

In this section, we use a query adapted from Coffman's benchmark over IMDb: "Which movies did Denzel Washington starred?". The initial process generated the SPARQL query below, ranking the results by the InfoRank score.

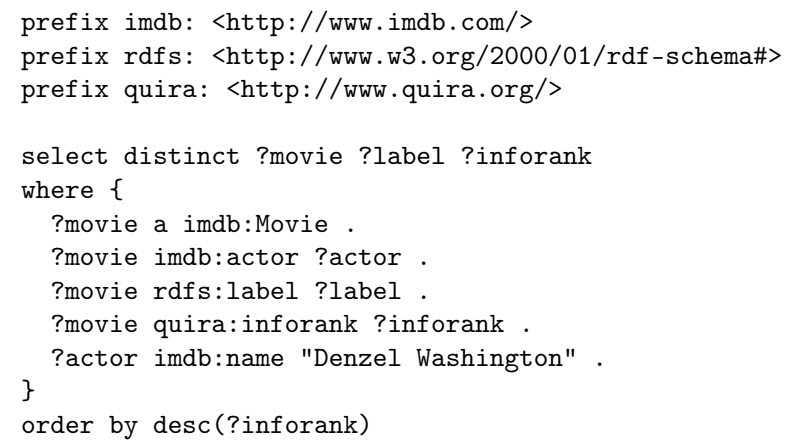

Table IX shows a preview of the original result. Note that this SPARQL query returned 49 movies starred by Denzel Washington. Again, we used the maximum of $\beta=15$ lines as the threshold to 
Table VI. Available predicates in the second reformulation process.

\begin{tabular}{|c|l|c|}
\hline$\#$ & Predicate & Distinct Values \\
\hline 1 & http://dbpedia.org/ontology/birthDate & 40 \\
\hline 2 & http://xmlns.com/foaf/0.1/givenName & 35 \\
\hline 3 & http://xmlns.com/foaf/0.1/name & 31 \\
\hline 4 & http://www.w3.org/2000/01/rdf-schema\#label & 27 \\
\hline 5 & http://dbpedia.org/ontology/wikiPageID & 26 \\
\hline 6 & http://dbpedia.org/ontology/wikiPageRevisionID & 26 \\
\hline 7 & http://purl.org/dc/terms/description & 20 \\
\hline 8 & http://dbpedia.org/ontology/activeYearsStartYear & 18 \\
\hline 9 & http://xmlns.com/foaf/0.1/surname & 16 \\
\hline 10 & http://dbpedia.org/property/caption & 13 \\
\hline 11 & http://dbpedia.org/property/occupation & 10 \\
\hline 12 & http://xmlns.com/foaf/0.1/gender & 2 \\
\hline
\end{tabular}

Table VII. Options for "Which of the above artist occupation do you prefer?".

\begin{tabular}{|l|l|c|}
\hline$\#$ & Options & Counts \\
\hline 1 & Singer-songwriter, musician & 5 \\
\hline 2 & Singer & 2 \\
\hline 3 & Musician & 1 \\
\hline 4 & Singer, actor & 1 \\
\hline 5 & Musician, songwriter & 1 \\
\hline 6 & Singer-songwriter & 1 \\
\hline 7 & Singer, author, philanthropist, actress & 1 \\
\hline 8 & Musician, singer-songwriter, record label owner & 1 \\
\hline 9 & Singer-songwriter, musician, visual artist & 1 \\
\hline 10 & Singer, rapper, songwriter, record producer, actor, businessman & 1 \\
\hline 11 & Show me all artists with some occupation & 15 \\
\hline 12 & Show me all artists & 27 \\
\hline
\end{tabular}

Table VIII. The final result presented to the user.

\begin{tabular}{|c|l|l|}
\hline$\#$ & Artist partial URI & Artist name \\
\hline 1 & /artist/a0580131-73f3-49c8-aac5-2c478f64a363 & Stephen Duffy \\
\hline 2 & /artist/19e07fd0-5642-47a0-a2b9-b8176e6b06e5 & Brooke Waggoner \\
\hline 3 & /artist/23c738ed-5dc4-4ff7-8c00-3c1c54e8eb89 & Kevin Barnes \\
\hline 4 & /artist/1d566a14-4094-4f96-abb7-969b4f439728 & Geva Alon \\
\hline 5 & /artist/4e0e884d-099b-4ca9-bf4d-bcb31e739540 & Duffy \\
\hline
\end{tabular}

indicate when the process should be applied. In this example, it would be a list with a maximum of $\beta$ movies. Hence, the process reformulated the SPARQL query to capture the predicates used for aggregation.

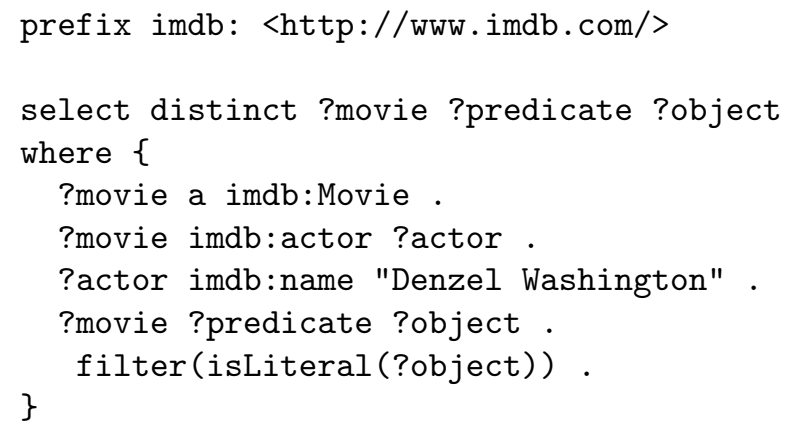

Once again, the process grouped the results by predicate and counted the distinct object values. 
Table IX. Preview of the original SPARQL result.

\begin{tabular}{|c|l|l|}
\hline$\#$ & Movie URI & Movie title \\
\hline 1 & http://www.imdb.com/work/1996688 & Malcolm X \\
\hline 2 & http://www.imdb.com/work/1592464 & American Gangster \\
\hline 3 & http://www.imdb.com/work/2354723 & Unstoppable \\
\hline$\ldots$ & $\ldots$ & $\ldots$ \\
\hline 47 & http://www.imdb.com/work/1675254 & Champs \\
\hline 48 & http://www.imdb.com/work/2255730 & The Equalizer \\
\hline 49 & http://www.imdb.com/work/2356601 & Uptown Saturday Night \\
\hline
\end{tabular}

Table X presents part of the available predicates. Following the proposed heuristic, the process chose the predicate imdb:label, which refers to the production company of the film. Hence, the process formulated the query "Which of the above movie label do you prefer?" and presented a few options for the user to choose from, as shown in Table XI.

Note that Table XI provides 12 options to the user: the first ten options refer to the movie label; the $11^{\text {th }}$ option to all movies with some label defined (not all movies have the label information defined in the database); and the last option to all movies (movies, without any filter). Suppose the user chose option "1. Columbia/Tristar" film label, which has one of the highest movie counts. The final result had only five movies, and the process stopped at this point since it achieved a reasonably compact result to present to the user. The final result was presented to the user in decreasing order of InfoRank score, as shown in Table XII.

Table X. Available predicates.

\begin{tabular}{|c|c|c|}
\hline$\#$ & Predicate & Distinct Values \\
\hline 1 & http://www.imdb.com/tag & 2408 \\
\hline 2 & http://www.imdb.com/release_dates & 1211 \\
\hline 3 & http://www.imdb.com/quotes & 936 \\
\hline$\ldots$ & $\ldots$ & $\ldots$ \\
\hline 36 & http://www.imdb.com/novel & 14 \\
\hline 37 & http://www.imdb.com/label & 10 \\
\hline 38 & http://www.imdb.com/number_of_chapter_stops & 8 \\
\hline$\ldots$ & $\ldots$ & $\ldots$ \\
\hline 66 & http://www.imdb.com/interviews & 1 \\
\hline 67 & http://www.imdb.com/master_format & 1 \\
\hline 68 & http://www.imdb.com/quality_program & 1 \\
\hline
\end{tabular}

Table XI. Options for "Which of the above movie label do you prefer?"

\begin{tabular}{|c|l|c|}
\hline$\#$ & Options & Counts \\
\hline 1 & Columbia/Tristar & 5 \\
\hline 2 & Encore & 5 \\
\hline 3 & Warner Home Video & 3 \\
\hline 4 & MCA/Universal Home Video & 2 \\
\hline 5 & Paramount & 2 \\
\hline 6 & 20th Century Fox Home Entertainment & 1 \\
\hline 7 & Hollywood Pictures & 1 \\
\hline 8 & Philips & 1 \\
\hline 9 & Pioneer & 1 \\
\hline 10 & RCA/Columbia & 23 \\
\hline 11 & Show me all movies with some label & 47 \\
\hline 12 & Show me all movies & \\
\hline
\end{tabular}


Table XII. Final result presented to the user.

\begin{tabular}{|l|l|l|}
\hline$\#$ & Movie URI & Movie title \\
\hline 1 & http://www.imdb.com/work/2095826 & Philadelphia \\
\hline 2 & http://www.imdb.com/work/1824613 & Glory \\
\hline 3 & http://www.imdb.com/work/2031102 & Much Ado About Nothing \\
\hline 4 & http://www.imdb.com/work/2020598 & Mississippi Masala \\
\hline 5 & http://www.imdb.com/work/1731607 & Devil in a Blue Dress \\
\hline
\end{tabular}

\subsection{Qualitative Discussion}

In this section, we discuss the results obtained for MusicBrainz and IMDb, from a qualitative point of view. We adopted a metric, called compression rate, to help compare the results obtained. The metric is denoted $\gamma$ and defined as follows:

$$
\gamma=1-\kappa / \eta
$$

where $\eta$ is the number of lines of the initial result set and $\kappa$ is the number of lines of the final result set.

Note that, when $\kappa \sim \eta$, the compression is very low. On the other hand, when $\kappa<<\eta$, the compression is very high. It is important to recall that $\beta$ guarantees that the final result set will not exceed a maximum number of lines. The analysis that follows will reflect this fact when the selected facet does not significantly reduce the result set.

Positioned at the end of this section, Tables XIV and XV have the same headers. Column IRS means Initial Result Set and is related to the number of lines from the original result set. Column FRS means Final Result Set and is related to the number of lines from the compressed result set. Column Facets has the selected predicate and facet separated by the pipe symbol "|". Note that the number between parenthesis is the number of unique subjects or, in other words, the number of lines of the result set filtered by facet. Also, this column may have multiple facets displayed in multi-lines. The number of lines reflects the number of Steps the process took.

Table XIV presents the results for MusicBrainz and lists seven questions. Recall that ten questions were originally tested over MusicBrainz, but only 3 of them had no predicate selected. This happened because the process could not find a predicate that respects the thresholds $\alpha=10$ and $\delta=(0.4,2.0)$ defined for the experiments. For instance, the question "What are the songs performed by Aretha Franklin?" has the following predicates: mo:track_number (54), rdfs:label $(1,120)$, and mo:duration $(1,881)$. In these cases, all three numbers in parenthesis are higher than $\alpha=10$. The original result set had 2,945 lines, and the final result set had $\beta=15$ lines. Finally, we recall that, even when the process does not perform any steps, it ranks the results using the InfoRank metric and returns the first $\beta$ lines to the user.

In the music context, it might be interesting to group songs by minutes. Based on the Music Ontology Specification ${ }^{5}$, the predicate mo:duration represents the duration of a track or a signal in ms. Analyzing the songs by Aretha Franklin, her longest song is "Amazing Grace" with 10min and 48s ( $\sim 11 \mathrm{~min})$. So, we can guarantee that the heuristic would apply one of the ten possible length-facets $(2 \mathrm{~min}-11 \mathrm{~min})$.

Continuing the analysis, the third question "Which artists played on the same groups that David Bowie was a member of?" had the original result set with length 17 , which is very close to $\beta=$ 15. Thus, the compression rate obtained by applying the heuristic was surprisingly large. But the predicate dbo: wikiPageID does not seem very interesting, considering a non-technical user. Hence,

\footnotetext{
${ }^{5}$ http://musicontology.com/specification/\#term-duration, (accessed 12 August 2020). 
the compression rate metric by itself does not capture all aspects. We still need to analyze the path explored by the process.

Table XV presents the results for IMDb and also lists seven questions. In this case, the most interesting predicates chosen and the facets selected were for the first question "Which movies did Denzel Washington starred?". Although the compression rates were good, when compared to the other questions, the compression rates were the worst (together with the sixth question). This happened because all other questions had no restrict facet and the original result set was very long. Thus, $\kappa<<\eta$ since $\kappa=\beta=15$.

In this specific scenario, the use of a ranked list of results is important, as otherwise the process would randomly choose $\beta$ results and return them to the user. Our process uses InfoRank to guarantee meaningful results. In Table XIII, the intersection between the results randomly sorted and the results sorted by InfoRank represents only $\sim 27 \%$ of $\beta=15$ movies. For instance, the multi-award-winning movie "Gladiator" would be missing in the result set.

Table XIII. Results comparison for "Which movies were released in 2000?".

\begin{tabular}{|c|c|c|c|c|}
\hline & \multicolumn{2}{|c|}{ Results randomly sorted } & \multicolumn{2}{|c|}{ Results sorted by InfoRank } \\
\hline \# & Movie partial URI & Movie title & Movie partial URI & Movie title \\
\hline 1 & /work/1637423 & Big Momma's House & /work $/ 2007705$ & Me, Myself \& Irene \\
\hline 2 & /work $/ \mathbf{2 3 9 3 8 8 3}$ & X-Men & /work/1823621 & Gladiator \\
\hline 3 & /work $/ \mathbf{1 6 2 5 8 2 2}$ & Battle Royale & /work $/ 2289533$ & The Patriot \\
\hline 4 & /work/1592761 & American Psycho & /work/2393883 & X-Men \\
\hline 5 & /work $/ 1551278$ & 2001: A Space Travesty & /work/1625822 & Battle Royale \\
\hline 6 & /work/1790475 & Faust: Love of the Damned & /work/2333414 & Traffic \\
\hline 7 & /work $/ 2363075$ & Versus & /work/1592761 & American Psycho \\
\hline 8 & /work/1812463 & Före stormen & /work/1676655 & Charlie's Angels \\
\hline 9 & /work $/ 2234180$ & The Adventures of Rocky \& Bullwinkle & /work $/ 2389882$ & Crouching Tiger, Hidden Dragon \\
\hline 10 & /work/2016980 & Militia & /work $/ 2133745$ & Requiem for a Dream \\
\hline 11 & /work/2350711 & Unbreakable & /work $/ 1685528$ & Citizen Toxie: The Toxic Avenger IV \\
\hline 12 & /work/1976757 & Little Nicky & /work/2061093 & O Brother, Where Art Thou? \\
\hline 13 & /work/2333414 & Traffic & /work $/ 2158237$ & Scary Movie \\
\hline 14 & /work/1807398 & Frequency & /work/2019684 & Miss Congeniality \\
\hline 15 & /work/2008819 & Meet the Parents & /work $/ 2139796$ & Road Trip \\
\hline
\end{tabular}

Finally, we investigated the lack of predicates with restrictive facets. Since the questions were related to imdb:Actor and imdb:Actress, we combined the sets of resources from imdb:Actor and imdb: Actress and called then Artists.

Analyzing the unique predicates related to instances of imdb:Movie, from 68 predicates, only 15 satisfy the $\alpha$ threshold. Also, considering the $\delta$ threshold, the set of selectable predicates is small, and their expressiveness is also low. Except for imdb: category, all selectable predicates were related to technical information about the movies. A similar scenario was observed for predicates related to instances of Artists. From the 26 available predicates, only one was selectable, regarding the defined thresholds.

\section{CONCLUSIONS}

The main contribution of this study was the definition of a process, called the Query Answer Modification Process, based on simple heuristics and parameterized thresholds, that analyses the query answer and improves the quality of the user's experience. This study addressed open-ended questions since specific questions do not generate long result sets, and would therefore not benefit from the summarization process.

To validate the proposed process, sample queries from the QALD challenge and Coffman's benchmark were used. These queries were applied over RDF versions of MusicBrainz and IMDb, respectively. 
Table XIV. Compression Rate comparison over Music Brainz.

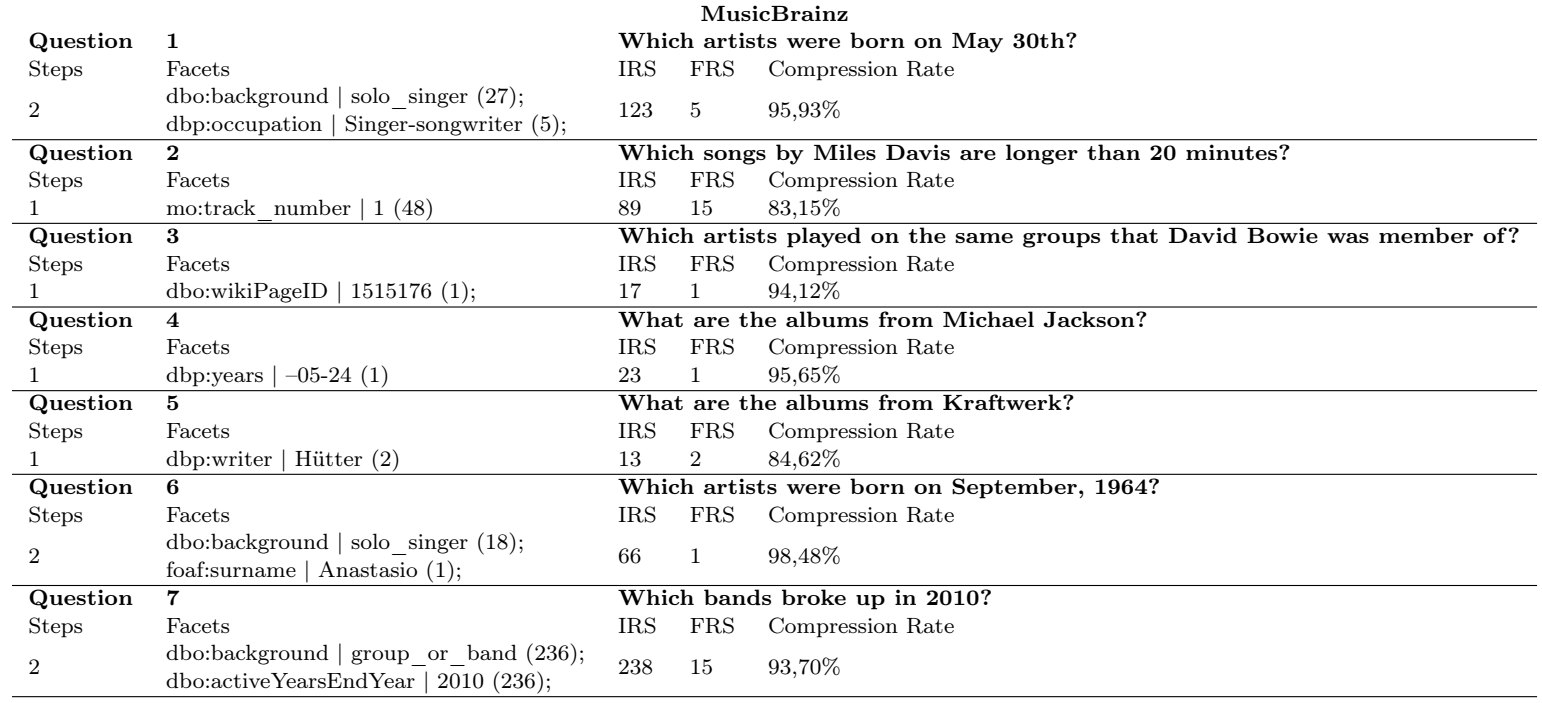

Table XV. Compression Rate comparison over IMDb.

\begin{tabular}{|c|c|c|c|c|}
\hline Question & 1 & \multicolumn{3}{|l|}{ IMDb } \\
\hline Steps & Facets & IRS & FRS & Compression Rate \\
\hline 1 & imdb:label | Columbia/Tristar (5); & 49 & & $89,80 \%$ \\
\hline Question & 2 & \multicolumn{3}{|c|}{ Which movies are available in spanish language? } \\
\hline Steps & Facets & IRS & FRS & Compression Rate \\
\hline 1 & imdb:color_info | Color (67933); & 77670 & 15 & $99,98 \%$ \\
\hline Question & 3 & \multicolumn{3}{|c|}{ Which actors or actresses were born on May 30th? } \\
\hline Steps & Facets & IRS & FRS & Compression Rate \\
\hline 1 & imdb:gender | Male (409); & 595 & 15 & $97,48 \%$ \\
\hline Question & 4 & \multicolumn{3}{|c|}{ Which movies were released in $2000 ?$} \\
\hline Steps & Facets & IRS & FRS & Compression Rate \\
\hline 2 & $\begin{array}{l}\text { imdb:color_info | Color (5815); } \\
\text { imdb:year } \mid 2000 \text { (5815); }\end{array}$ & 7206 & 15 & $99,79 \%$ \\
\hline Question & 5 & \multicolumn{3}{|c|}{ Which movies were produced in Brazil? } \\
\hline Steps & Facets & IRS & FRS & Compression Rate \\
\hline 1 & imdb:color_info | Color (4432); & 7016 & 15 & $99,79 \%$ \\
\hline Question & 6 & \multicolumn{3}{|c|}{ Which movies were produced in Brazil in 2010? } \\
\hline Steps & Facets & IRS & FRS & Compression Rate \\
\hline 2 & $\begin{array}{l}\text { imdb:color info | Color (74); } \\
\text { imdb:year | } 2000(74)\end{array}$ & 91 & 15 & $83,52 \%$ \\
\hline Question & 7 & \multicolumn{3}{|c|}{ Which Brazilian artists starred foreign movies? } \\
\hline Steps & Facets & IRS & FRS & Compression Rate \\
\hline 1 & imdb:gender | Male (733); & 1261 & 15 & $98,81 \%$ \\
\hline
\end{tabular}

Also, a compression rate metric was defined, enabling a comparison and discussion over the compiled results.

The first suggestion for future work would be to allow users to provide a list of predicates to ignore or to enable them to dynamically exclude undesired predicates from the available list in each step of the process. Thus, it would be possible to exclude predicates without a clear contribution, from the user's point of view, and the results would possibly improve. Also, in this context, registering users' feedback after each query answer modification would provide a strategy to construct this list automatically.

A second suggestion would be to develop and apply a questionnaire to users interested in using the system. As a result, we would be able to segment users by preferences and create user profiles. These profiles would enrich the qualitative discussion in Section 4 and could be used to adapt the heuristics to the users' preferences. 
Another interesting suggestion would be to allow users to indicate that specific predicates would actually define a taxonomy so that the system would be able to aggregate over each level of the taxonomy. In a second version, together with the initial exploration of the RDF Knowledge Base to calculate frequencies, the system would automatically infer the related taxonomy.

Finally, it would be profitable to develop a user interface similar to GraFa [Moreno-Vega and Hogan 2018] which would allow navigation through predicates and facets. An extra feature that would impact the user's navigation is the possibility to select more than one option at each interaction. Hence, disjunctive filtering would be possible, together with conjunctive filtering, as defined in this article.

\section{ACKNOWLEDGMENT}

This work was partly funded by the Coordenação de Aperfeiçoamento de Pessoal de Nível Superior Brasil (CAPES) - Finance Code 001, and grants CAPES 88881.134081/2016-01, CNPq 302303/2017-0 and FAPERJ E-26-202.818/2017.

\section{REFERENCES}

Coffman, J. And Weaver, A. C. A framework for evaluating database keyword search strategies. In Proceedings of the 19th ACM international conference on Information and knowledge management. pp. 729-738, 2010.

Cyganiak, R., Wood, D., And Lanthaler, M. RDF 1.1 Concepts and Abstract Syntax, 2014. W3C Recommendation 25 February 2014.

Dalianis, H. And Hovy, E. Aggregation in natural language generation. In Trends in Natural Language Generation An Artificial Intelligence Perspective, J. G. Carbonell, J. Siekmann, G. Goos, J. Hartmanis, J. Leeuwen, G. Adorni, and M. Zock (Eds.). Vol. 1036. Springer Berlin Heidelberg, Berlin, Heidelberg, pp. 88-105, 1996.

Diefenbach, D., Lopez, V., Singh, K., And Maret, P. Core techniques of question answering systems over knowledge bases: a survey. Knowledge and Information Systems 55 (3): 529-569, June, 2018.

Diefenbach, D., Tanon, T. P., Singh, K., And Maret, P. Question Answering Benchmarks for Wikidata. In ISWC 2017. Vienne, Austria, 2017.

Franz, T., Schultz, A., Sizov, S., and StaAb, S. TripleRank: Ranking semantic web data by tensor decomposition. In Lecture Notes in Computer Science. Springer Berlin Heidelberg, Berlin, pp. 213-228, 2009.

Menendez, E. S., Casanova, M. A., Leme, L. A. P., and Boughanem, M. Novel node importance measures to improve keyword search over rdf graphs. In International Conference on Database and Expert Systems Applications. Springer, pp. 143-158, 2019.

Moreno-Vega, J. And Hogan, A. Grafa: Faceted search \& browsing for the wikidata knowledge graph. In International Semantic Web Conference. Springer, Cham, 2018.

Novello, A. And Casanova, M. A. A novel solution for the aggregation problem in natural language interface to databases (nlidb). In Anais do XXXV Simpósio Brasileiro de Bancos de Dados. SBC, Porto Alegre, RS, Brasil, pp. $217-222,2020$.

Petzka, H., Stadler, C., Katsimpras, G., Haarmann, B., and Lehmann, J. Benchmarking faceted browsing capabilities of triplestores. In Proceedings of the 13th International Conference on Semantic Systems. Semantics2017. Association for Computing Machinery, New York, NY, USA, pp. 128-135, 2017.

Prud'hommeaux, E. And Seaborne, A. SPARQL Query Language for RDF, 2008. W3C Recommendation 15 January 2008.

WebBer, B. L. Questions, answers and responses: Interacting with knowledge-base systems. In Topics in Information Systems. Springer New York, pp. 365-402, 1986.

Wei, B., Liu, J., Zheng, Q., Zhang, W., Fu, X., And Feng, B. A survey of faceted search. Journal of Web Engineering vol. 12, pp. 41-64, 02, 2013. 